\title{
Psychological performance assessment via interactive voice response systems
}

\author{
JAMES C. MUNDT and PHILIP F. KELLEHER \\ Vermont Alcohol Research Center, Colchester, Vermont \\ and \\ M.W. PERRINE and JOHN S. SEARLES \\ Vermont Alcohol Research Center, Colchester, Vermont \\ and University of Vermont, Burlington, Vermont
}

\begin{abstract}
Two experiments examined the feasibility of psychological assessment using interactive voice response (IVR) technology and the potential sensitivity of such assessments to alcohol and fatigue effects. In Experiment 1, 10 subjects performed a 12-min battery of six IVR-administered tasks, Monday through Friday, over 2 weeks. Minimal learning effects were evident during training. Repeated administrations indicated high test-retest reliabilities. In Experiment 2 (double-blind, alcohol/placebo crossover design), 7 subjects were tested every $2 \mathrm{~h}$ over a 24 -h period during two experimental sessions (peak blood alcohol concentrations $=80 \mathrm{mg} / \mathrm{dL}$ ). Several IVR-administered tasks were sensitive to alcohol impairment, but not as sensitive as laboratory-based measures specifically designed to assess alcohol impairment. Little evidence for fatigue-related impairment was obtained. The results support optimism for the potential to assess psychomotor and cognitive functioning distally via telephony; however, further refinement and validation of the methods are needed.
\end{abstract}

The burgeoning growth of interactive voice response (IVR) applications - automated telephone attendants that accept touch-tone telephone responses - over the past decade attests to the flexibility and utility of these systems. IVR applications for bank account status, airline schedules, and college course registration are now commonplace. IVR systems for routing incoming telephone calls to specific departments and individuals and maintaining voice messaging systems are becoming increasingly pervasive in government and business organizations.

More recently, research-oriented IVR applications have been developed that integrate computerized interview and longitudinal survey methodologies. In contrast to prior IVR applications designed to provide information and service to callers, these applications have been designed to obtain information from callers in order to address specific behavioral research questions. Computerized applications for obtaining psychiatric diagnostic information have been validated and used for decades (e.g., Greist, Gustafson, et al., 1973; Greist \& Klein, 1980; Greist, Klein, \& Van Cura, 1973; Greist, Van Cura, \& Kneppreth, 1973; Richards, Fine, Wilson, \& Rogers, 1983) and have recently begun to be

This research was supported by National Institute on Alcohol Abuse and Alcoholism Grant P01 AA07203 to M. W. P. The IVR tasks were programmed and the resulting database managed by Dan Walter. The authors thank Elizabeth Chamberlain, Donal Forgays, Mike George, Carroll Guitar, Gabrielle Kellogg, Chris Lussier, Pam McGonagleMoulton, Will McGrath, Cindy Proulx, and C. J. Rairikar for their participation in the first experiment. Correspondence should be addressed to J. C. Mundt, who is now at the Dean Foundation for Health Research and Education, 2711 Allen Blvd., Middleton, WI 53562 (e-mail: mundt james_c@ssmhes.com). implemented in IVR applications (Baer, Brown-Beasley, Sorce, \& Henriques, 1993; Baer et al., 1995). Prospective longitudinal studies of self-reported daily alcohol consumption using an IVR system have been conducted (Mundt, Perrine, Searles, \& Walter, 1995; Searles, Perrine, Mundt, \& Helzer, 1995), providing insight into the consequences and clinical significance associated with different drinking patterns (Mundt, Searles, Perrine, \& Helzer, 1995). Such applications now represent an established, validated longitudinal research method (Perrine, Mundt, Searles, \& Lester, 1995), and extensions to other behavioral aspects of daily life are being developed.

Data obtained via IVR for research purposes, to date, have been supplied exclusively as self-report in response to specific questions. Given the successful implementation of IVR applications for psychiatric assessment, it is feasible that IVR applications might be developed to assess other aspects of psychological functioning. There are now scores of computer-based performance assessment tools available commercially (Psychological Corp., 1996); researchers continue to develop tests that can be obtained at little or no cost (e.g., Elsmore, 1994; McKnight \& McKnight, 1994). The development, characteristics, and desired properties of such tools have been discussed in the literature (e.g., Turnage \& Kennedy, 1992; Turnage, Kennedy, Smith, Baltzley, \& Lane, 1992). Test characteristics such as measurement stability, task definition, reliability, and performance stabilization time are critical factors influencing the acceptability and utility of such tests, particularly for repeated administration of the tests over time. Test stabilization is addressed through examination of group means and standard deviations; differential sta- 
bility, task definition, and reliability are evaluated with respect to intertrial correlations of measured performance (Kennedy, Baltzley, Wilkes, \& Kuntz, 1989). The validity of such tests can be assessed only with respect to (1) convergent results obtained with previously validated instruments, (2) predictive accuracy for future performance, and (3) the face validity of the tasks putatively tapping psychological domains determining measured performance.

Most computer-based performance assessment batteries present information to subjects visually, via monitors, although auditory tasks have been included in applications designed to assess multitask performance abilities. Present limitations of widely available telephone systems require that IVR-based assessment batteries rely exclusively on auditory presentation of information, although technological advances incorporating screen-based interfaces are being developed. Auditory presentation of information constrains the breadth of psychological assessment that is currently feasible; however, many aspects of cognitive abilities requiring the encoding and processing of information and the execution of appropriate responses are assessable through either visual or auditory presentation of information.

This paper describes two experiments designed to ascertain the feasibility and potential for implementing IVR-administered tasks to assess psychological functions. Experiment 1 investigated task stabilization parameters with respect to acquisition of asymptotic performance levels over a 1-h training session and the differential sensitivity of the tasks to reflect individual differences in performance ability over a 2-week interval. Experiment 2 investigated the sensitivity and validity of these tasks for assessing fatigue and alcohol-induced impairment.

\section{EXPERIMENT 1}

\section{Method}

\section{Subjects}

Five men and 5 women, ranging from 25 to 48 years old, served as subjects. All of the subjects were employees of the Vermont Alcohol Research Center and volunteered to participate in the experiment. They were not compensated for their participation.

\begin{abstract}
Apparatus
Administration of tasks was controlled by an application program written in EASE (Expert Systems Inc., Atlanta, GA) and implemented on a 486DX-based IVR system. Data inputs were captured using a Dialogic 4.1 voice board and stored by EASE in Microsoft FoxPro 2.5 relational database tables. Standard desktop Comdial Executech Model 6614T-FV telephones were used to generate the response inputs.
\end{abstract}

\section{IVR Psychological Assessment Battery}

The subjects performed a battery of six IVR-administered tasks designed to assess psychomotor and cognitive functioning. The tasks were educated guesses, derived from tasks described in existing literature regarding computer assessment of psychological functioning and the apparent similarity (face validity) of the tasks with established neuropsychological assessment instruments. The tasks were (1) follow the leader, (2) ordering, (3) one back, (4) digit span, (5) half task, and (6) memory probe. The tasks were always administered in this order. During the tasks, the subjects responded to au- ditory stimuli (numbers " 1 " through " 9 ") by pressing the $0-9$, \#, or * keys of a touch-tone telephone. Except where noted below, a 2-sec time-out interval was allowed for a response to be made before the next stimuli were automatically presented. The presentation time of each number was $400 \mathrm{msec}$, with an interstimulus interval of $300 \mathrm{msec}$ for multidigit sequence presentations (which terminated with a $100-$ msec tone). Total task duration for the follow-the-leader, ordering, one-back, and half tasks was $90 \mathrm{sec}$ each. Prior to each task, the subjects were instructed to work as quickly and accurately as possible.

Follow-the-leader task. The subjects heard the digits 1-9 randomly presented, one at a time, and responded to each digit by pressing the key corresponding to the digit. Performance was measured by the number of correct and incorrect keypresses during the 90 -sec task period.

Ordering task. The subjects heard three digits presented in sequence. Each sequence included one digit from each row of the telephone keypad (1-3), (4-6), and (7-9). The digits were presented in a random order. The subjects responded by pressing the keys corresponding to the presented digits in ascending order (top row, middle row, bottom row). Thus, the order of keypress responses was not necessarily the same as the stimulus set presentation order. The subjects were instructed to terminate their three-digit response by pressing the \# key. Failure to press the \# key did not constitute an error, but required the subject to wait idly through a 4-sec time-out interval. Thus, use of the \# key permitted rapid progression from one sequence to the next. Performance was measured by the number of correct and incorrect sequences entered during the 90 -sec task period.

One-back task. The subjects heard random digits (1-9) presented one at a time, after an initial presentation of two digits. The subjects responded to each digit by pressing the key corresponding to the digit presented prior to the most recently presented digit. For example, if a " 7 " was presented following a " 3 ," the correct response was to press the $3 \mathrm{key}$; the correct response following the next digit was the $7 \mathrm{key}$, regardless of the next digit presented. The subjects were instructed to use a press-and-move strategy during training: "When the first digit is presented, place your finger on that key; for each subsequent digit, press the key under your finger and then move your finger to the key corresponding to the new digit presented." Performance was measured by the number of correct and incorrect keypresses during the 90 -sec task period.

Digit-span task. The subjects heard random digit sequences and responded by pressing the keys corresponding to the digits in the sequence in the same order, followed by the \# key. The \# key functioned similarly to the \# key in the ordering task. Each trial began with a single-digit sequence. Following a correct response to a presented sequence, the length of the subsequent sequence was increased by one digit. Each sequence presented was independent of preceding sequences (i.e., they did not build upon previously presented sequences). A trial terminated following either an errant response to a presented sequence or the successful completion of a $10-$ digit sequence. Digit-span performance was assessed five times in succession. Performance was measured by the length of the longest sequence successfully responded to in each of the five trials.

Half task. The subjects were presented with random digits (1-9). Similar to the one-back task, the digits were presented one at a time following an initial presentation of two digits. The subjects were required to press one of three keys following each digit presentation (starting with the second digit presented). The \# key was to be pressed if the presented digit was the same as the preceding digit, the ${ }^{*}$ key was to be pressed if the presented digit was one half of the preceding digit, or the key corresponding to the presented digit was to be pressed if neither of these conditions was true. For example, if " 4 " was presented, the correct response was \# if the previous digit had been " 4, , * if the previous digit had been " 8 ," or 4 if the previous digit had been other than " 4 " or " 8 ." The sequence of digits was pseudorandomized such that each type of response was required with equal frequency. Performance was measured by the number of correct and incorrect keypresses during a 90 -sec task duration. 
Memory-probe task. The subjects were presented with a fivedigit (1-9) memory set sequence. The digits were presented in random order with no repeated digits. The subjects were instructed to memorize the sequence and then press the \# key (8-sec time-out interval allotted for set memorization). The subjects were then presented a probe digit and asked to respond by pressing the key corresponding to the position of the probe in the memorized sequence or 0 if the probe was not in the sequence. For example, if presented with a memory set of "6 2851 " and a probe of " 5 ," the correct response would be 4 (fourth position). The subjects completed 12 such trials: 2 with the probe in each of the five possible sequence positions, and 2 for which the probe was not in the presented sequence. Performance was measured as the number of correct responses across the 12 trials.

\section{Procedure}

Each subject received $1 \mathrm{~h}$ of training on the IVR tasks within the 2 weeks prior to the start of the 2-week study. During the training session, the IVR task battery was administered four times in succession. The subjects were instructed how to access the IVR system and enter subject identification information.

The subjects called the IVR system once each day between 9 a.m. and 4 p.m., Monday through Friday, over a 2-week period. After entering subject identification information, the six tasks of the IVR test battery were administered. Brief instructions were provided prior to the administration of each task. Approximately 12 min were needed to complete the battery of tasks each day.

\section{Results}

Initial concerns regarding the data obtained by IVRadministered tasks focused on the amount of training needed for the subjects to acquire stable levels of task performance and the reliability of the task measures to discriminate individual differences in performance ability. The performance measures obtained for each of the tasks during training were analyzed in analyses of variance (ANOVAs), with gender as a grouping variable and the four training trials as a repeated measure. Gender was not a significant factor in any of the analyses, nor did it interact across trials. The group mean performance and standard deviations for the six tasks over the four training trials, collapsed over gender, are shown in Figure 1.

The results of the ANOVAs indicated that learning effects were minimal or nonexistent, producing stable task performance quite rapidly. For the digit-span, memoryprobe, and follow-the-leader tasks, there was virtually no improvement in performance as a function of practice $\left[F_{\mathrm{S}}(3,24)=0.43,0.71\right.$, and 0.71 , respectively, all $\left.p \mathrm{~s}>.5\right]$. The improvement in performance over trials in the ordering task was marginally significant $[F(3,24)=2.48, p=.09$; linear component, $F(1,8)=5.36, p<.05]$, and the effect of training trials was significant in the one-back and half tasks $[F \mathrm{~s}(3,24)=7.69$ and $18.09, p \mathrm{~s}<.001$, respectively $]$. Post hoc $t$ tests comparing performances from successive trials indicated significant improvement between Training Trials 1 and 2 for both the one-back task $[t(9)=5.13, p<$ $.001]$ and the half task $[t(9)=5.03, p<.001]$. No other differences between successive training trials approached statistical significance.

The degree to which individual differences were reliably evident in the training data was assessed by analyzing the Spearman rank-order correlations of the performances obtained during each training trial with the performances obtained on the prior trial. Rank-order correlations were used to assess the test-retest reliabilities due to the relatively small sample size. These data are provided in Table 1 (asterisks indicate correlations significant at $\alpha=.05$ ).

The group mean performance and standard deviations for the six tasks across the 10 days of the 2-week study are shown in Figure 2. All of the data from follow-the-leader task on the lst day of the experiment were lost due to a data storage problem, and data from the memory-probe and half tasks were lost for one of the subjects on the 9th day of the study due to a telephone hardware problem. The individual's mean performance on these tasks across the other nine study days was used to replace these two missing data points.

Repeated measures ANOVAs, including gender as a between-subjects variable, were computed on the performance measures obtained on each of the tasks across the 10 days of the study (the last 9 days for the follow-theleader task). Greenhouse-Geisser corrections were used to adjust the reported probability values, due to sphericity violations in the repeated measures error component. Gender was not a significant factor in any of the analyses, nor did it interact with performance across study days (all $p \mathrm{~s}>.05$ ). For the digit-span, memory-probe, follow-theleader, and one-back tasks, no significant effects across the study days were obtained $[F(9,72)=1.40, F(9,72)=1.96$, $F(8,64)=1.29$, and $F(9,72)=2.04$, respectively, all $p \mathrm{~s}>.1$. For the ordering and half tasks, the omnibus ANOVAs did indicate a significant effect over study days $[F(9,72)=$ $3.66, p<.05$, and $F(9,72)=9.31, p<.0001$, respectively]. Post hoc matched $t$ tests were computed between performances on consecutive days for these tasks to ascertain whether the significant effects were attributable to specific day-to-day variations in performance, or whether they reflected the generally upward trend in performance across the study duration evident in the graphs of Figure 2. There were no significant differences between consecutive study days in the ordering task data (all $p \mathrm{~s}>.05$ ), but analysis of the half task data indicated significant performance improvement from Day 3 to Day $4[t(9)=2.26, p=$ $.05]$ and from Day 9 to Day $10[t(9)=2.51, p<.05]$.

The consistency of individual day-to-day performance differences was also assessed by Spearman rank-order correlation because of the small sample size. Performances obtained on each of the study days were rank ordered and correlated with the rank ordering of the performances of the day before. Table 2 shows the rank-order correlations for each study day with respect to the performances obtained on the prior day. These values compare well with the data of Table 1, indicating slightly lower reliability for distinguishing individual differences in performance ability than the training data.

\section{Discussion}

The results of Experiment 1 are encouraging for the feasibility of implementing tasks designed to assess psychomotor and cognitive functioning via IVR. The data ob- 
Ordering Task
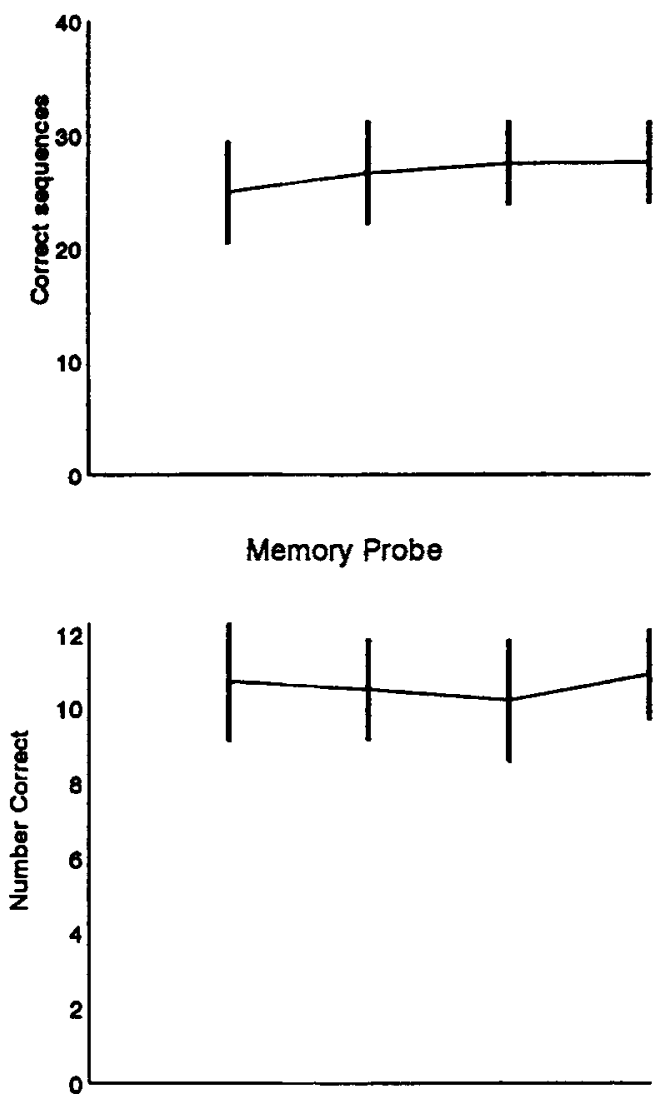

Digit Span

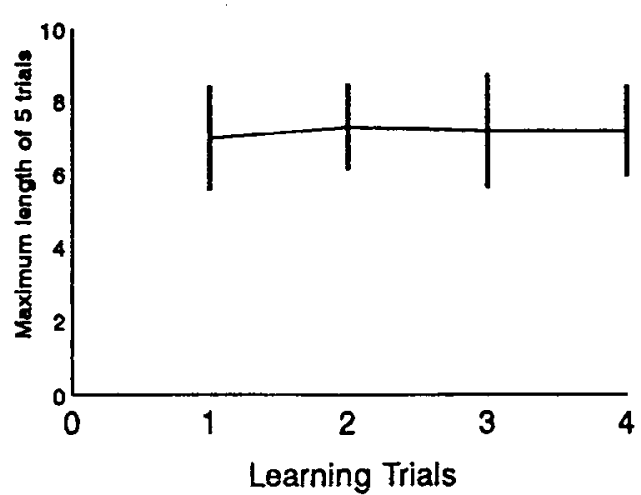

One Back Task

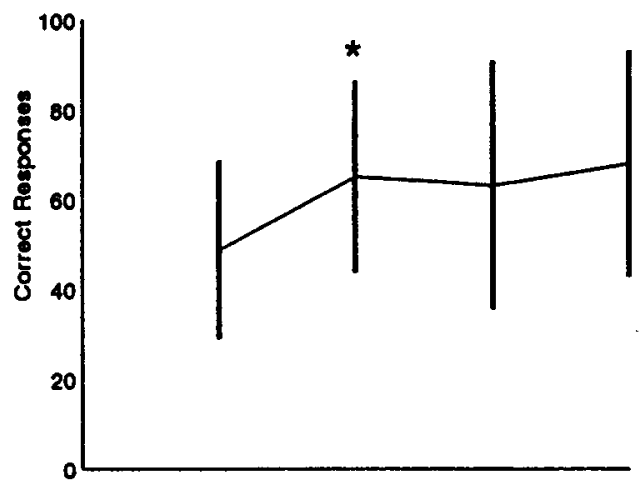

Follow the Leader

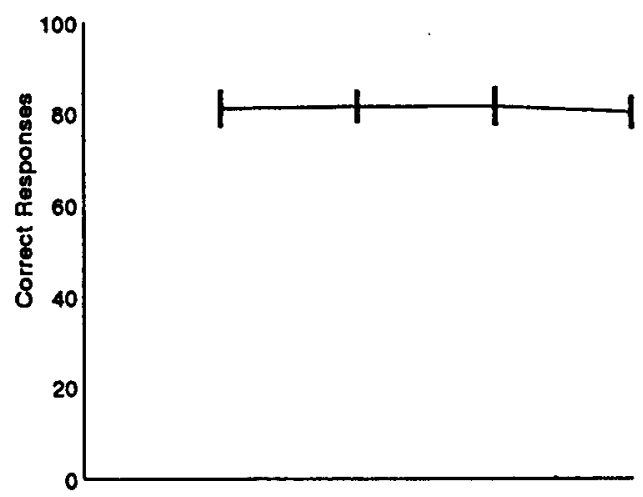

Half Task

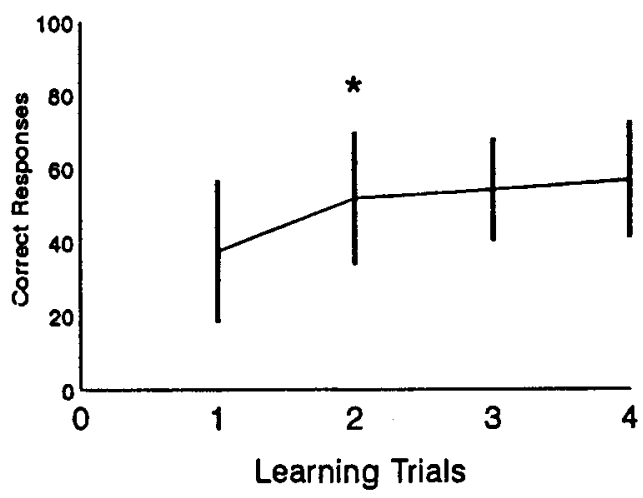

Figure 1. Mean performance for each of the six IVR administered tasks $( \pm 1 S D)$ acress the four training trials. No significant practice effect is indicated for memory-probe, follow-the-leader, or digit-span performance. The linear practice effect for the ordering task is significant $(p<.05)$, and the significant practice effects in the one-back and half tasks reflect the significant improvement between Training Trials 1 and 2 , indicated by asterisks.

tained during the training phase indicate that stable performance levels, sensitive to individual differences in ability, were obtained very quickly. For all but two of the tasks, performance was essentially asymptotic from the initial assessment. Stable performances were obtained on the one-back and half tasks after one practice trial. The evidence for rapid acquisition of asymptotic performance levels during training was diminished somewhat by the upward trend in performance over the 10 days of the study in the memory-probe, ordering, and half tasks; however, 
Table 1

Rank-Order Correlations for Each of the

Six Tasks Referencing Performances Obtained

by the 10 Subjects on the Prior Training Trial

\begin{tabular}{ccccccc}
\hline & \multicolumn{6}{c}{ Task } \\
\cline { 2 - 7 } Training Trial & $\begin{array}{c}\text { Digit } \\
\text { Span }\end{array}$ & $\begin{array}{c}\text { Follow } \\
\text { the Leader }\end{array}$ & Half & $\begin{array}{c}\text { Memory } \\
\text { Probe }\end{array}$ & One Back & Ordering \\
\hline 2 & $.71^{*}$ & $.60^{*}$ & $.76^{*}$ & .43 & $.89^{*}$ & $.75^{*}$ \\
3 & $.87^{*}$ & $.75^{*}$ & $.77^{*}$ & $.60^{*}$ & $.82^{*}$ & $.86^{*}$ \\
4 & $.91^{*}$ & $.84^{*}$ & $.94^{*}$ & .24 & $.87^{*}$ & $.72^{*}$ \\
Mean & .83 & .73 & .82 & .42 & .86 & .78 \\
\hline
\end{tabular}

*Statistically significant, $\alpha=.05$ (single-tailed test of positive correlation).

the continued rise in performance on these tasks as a function of experience was fairly small, relative to the differences between subjects in performance abilities.

Conservative test-retest reliabilities were assessed in this experiment using Spearman rank-order correlations between juxtaposed trials because of the limited sample size and the sensitivity of Pearson correlations to extreme outliers. Tables 1 and 2 clearly indicate that four of the six tasks reliably assess individual differences in performance ability. The other two tasks - the memory-probe and followthe-leader tasks - did not produce statistically significant reliability coefficients; however, this may reflect ceiling effects in the data. Performance on the memory-probe task was limited to a maximum of 12 correct responses; $41.3 \%$ of all administered trials obtained this score, and an additional $23.9 \%$ provided 11 correct responses. The overall average performance on the follow-the-leader task was 83.9 correct responses over the 90 -sec task duration, or 1 response every $1,073 \mathrm{msec}$. With each digit requiring $400 \mathrm{msec}$ to present, the subjects processed the information presented and executed a correct response among the 9 possible responses with an average reaction time of $673 \mathrm{msec}$. The mean standard deviation across all trials was 5.5 responses, representing an average reaction time difference of only $71 \mathrm{msec}$ per response, or less than $7 \%$ of the mean item response time (presentation plus reaction time). These considerations indicate that repeated measurements of the memory-probe and follow-theleader tasks produced reliable data that were not sensitive to individual differences in ability, because the tasks were being performed about as well as they could be. Performance on these tasks, however, may be influenced by impairment due to drugs, fatigue, or brain damage.

The results of Experiment 1 indicate that repeated performance assessment via IVR is feasible and produces reliable data. The data do not address whether measurements obtained in such a fashion are sensitive to subject impairment-a question addressed in Experiment 2.

\section{EXPERIMENT 2}

\section{Method}

\section{Subjects}

Seven men who had participated previously in other studies at the Vermont Alcohol Research Center (VARC) served as subjects. An 8th subject began the experiment but could not complete the protocol due to unanticipated transportation problems. The 7 subjects ranged from 28 to 40 years old $(M=33)$. All of the subjects were White; 1 was married, 2 had live-in partners, 1 was divorced, and 3 had never been married; 5 were employed full time, and the other 2 worked part time; all of the subjects had completed 12 or more years of schooling. With regard to alcohol consumption, 2 subjects reported drinking one to two times per week, 3 reported drinking three to four times per week, and 2 reported daily consumption. The subjects reported an average of 3.4 drinks per typical sitting, ranging from 2 to 5 drinks.

Treatment of the subjects was in accordance with the Ethical Principles in the Conduct of Research with Human Participants (American Psychological Association, 1989) and Recommended Council Guidelines on Ethyl Alcohol Administration in Human Experimentation (National Advisory Council on Alcohol Abuse and Alcoholism, 1989). The subjects were compensated $\$ 375$ for completing the experiment. The subjects were not allowed to drive to the sessions due to the anticipated fatigue. Transportation expenses were reimbursed.

\section{Apparatus}

Administration of the IVR tasks was the same as that used in Experiment 1 . Additional performance measures were obtained using a Model 30014 Photoelectric Rotary Pursuit (Lafayette Instrument Co., Lafayette, IN)-hereafter referred to as a pursuit rotor-and the simulated evaluation of drug impairment (SEDI) task (Profiles Associates, Chapel Hill, NC), a visual-based divided-attention/choice reaction-time task. Blood alcohol concentrations (BACs) were measured using Model 1400 Intoxilyzers (CMI, Inc., Owensboro, KY).

\section{Procedure}

Session schedule. All of the subjects participated in two testing sessions held on consecutive weekends. The subjects arrived for each session Friday evening at 9:30 p.m. On arrival, breath samples were obtained to ensure that initial BACs were 0 . Age, height, and weight data were then obtained for dosing calculations. After a short briefing session and procurement of informed consent, 4 of the subjects slept overnight in special laboratory facilities, and the others were provided rooms at a nearby hotel. The subjects were instructed to be in bed by 11 p.m.

The subjects were awakened Saturday at 6:30 a.m. From Saturday morning until session completion Sunday morning, the subjects completed all of the performance tasks 13 times. The testing trials were spaced at 2-h intervals. During each session, the subjects were divided into two groups. The first group started at 8 a.m. (subsequent trials began at 10 a.m., noon, 2 p.m., etc., until the final trial $24 \mathrm{~h}$ later at $8 \mathrm{a} . \mathrm{m}$. Sunday). The second group began the first trial at 9 a.m. and were lagged $1 \mathrm{~h}$ behind the first group for the remainder of the session. Scheduled drinking periods and meals are described below. After the final trial, the subjects were debriefed and released when their transportation arrived.

Performance assessment trials. BAC measurements were obtained at the beginning and end of each trial. During the trial, the subjects were rotated through three testing stations in which they 
Ordering Task

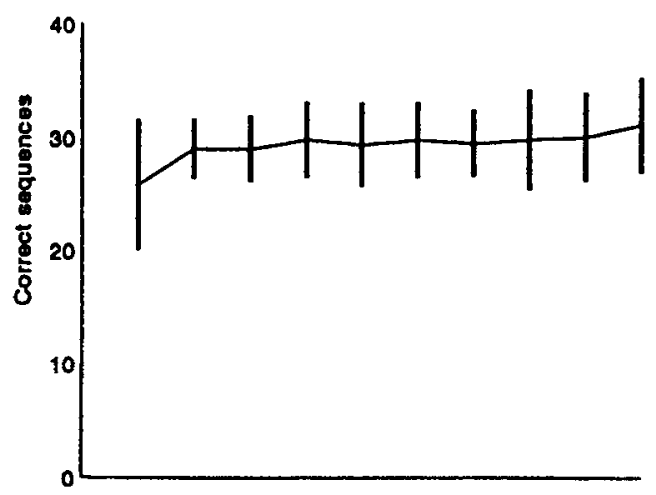

Memory Probe

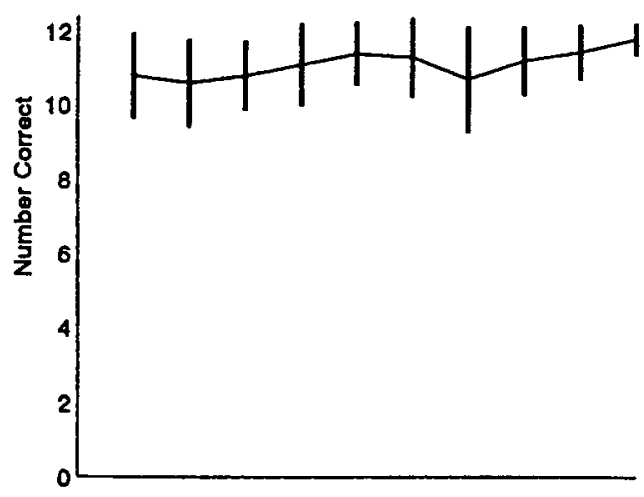

Digit Span

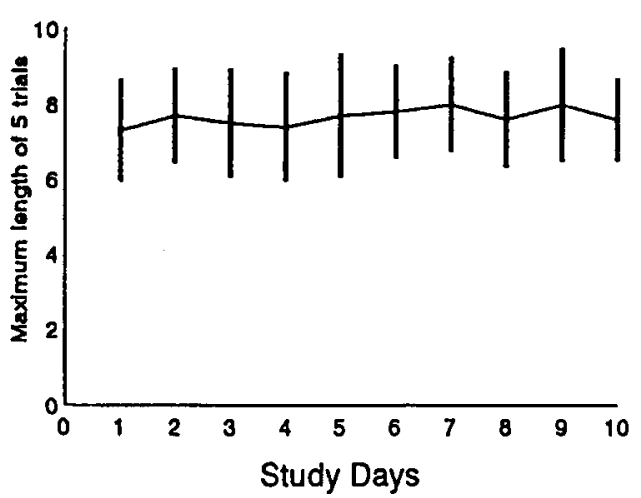

One Back Task

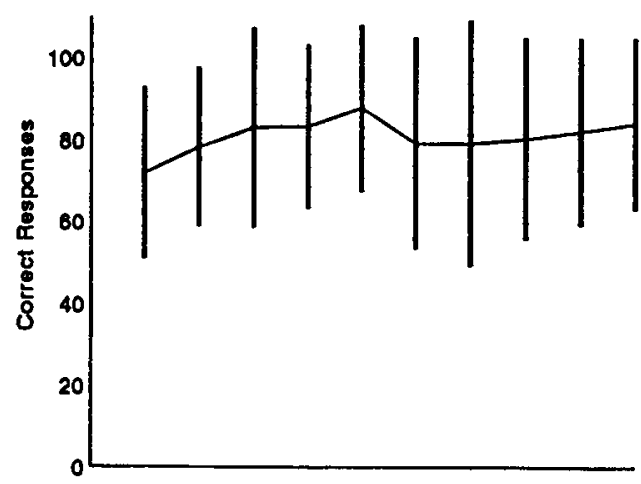

Follow the Leader

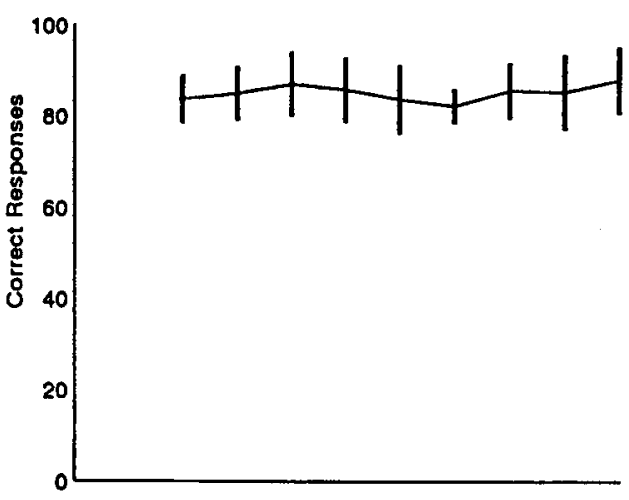

Half Task

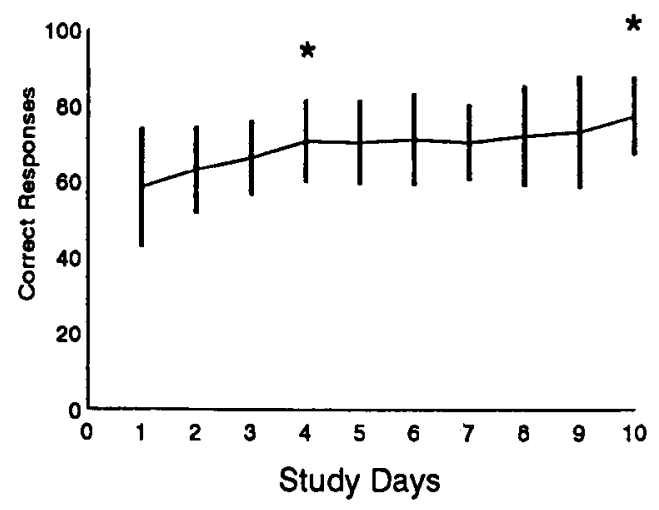

Figure 2. Mean performance for each of the six IVR administered tasks ( $\pm 1 S D$ ) across the 10 study days. No statistically significant effect is evident across study days for digit-span, follow-the-leader, or one-back performance. Significant main effects across study days for the memory-probe, ordering, and half tasks were analyzed with matched $t$ test between consecutive day performances. Significant day-to-day changes were found in the half task, indicated by the asterisks.

performed the IVR, pursuit-rotor, and SEDI tasks; a trial required approximately $30 \mathrm{~min}$ to complete all tasks. The IVR tasks were performed in the same order used in Experiment 1. The pursuit-rotor task consisted of two consecutive 50 -sec runs, during which a luminous target moved in a clockwise circle at a constant speed of $30 \mathrm{rpm}$. Performance was measured by the average time that the photosensitive stylus was above the target across the two runs. The use of the pursuit-rotor task in alcohol research is discussed in Vogel-Sprott (1992). The SEDI task required the subjects to monitor numbers appearing simultaneously on three displays and to execute one of five responses upon detection of predesignated stimulus values. Approximately $2 \mathrm{~min}$ were required to complete each SEDI trial, during which approximately 240 number changes occurred across the three displays. During each trial, 50 target stimuli were presented 
Table 2

Rank-Order Correlations for Each of the Six Tasks Referencing

Performances Obtained by the Subjects on the Prior Study Day.

\begin{tabular}{rcccccc}
\hline & \multicolumn{5}{c}{ Task } \\
\cline { 2 - 7 } Study Day & $\begin{array}{c}\text { Digit } \\
\text { Span }\end{array}$ & $\begin{array}{c}\text { Follow } \\
\text { the Leader }\end{array}$ & Half & $\begin{array}{c}\text { Memory } \\
\text { Probe }\end{array}$ & One Back & Ordering \\
\hline 2 & $.65^{*}$ & - & $.76^{*}$ & .35 & $.85^{*}$ & $.73^{*}$ \\
3 & .53 & .13 & $.75^{*}$ & $.62^{*}$ & $.61^{*}$ & $.78^{*}$ \\
4 & $.94^{*}$ & $.81^{*}$ & $.73^{*}$ & .27 & $.84^{*}$ & $.82^{*}$ \\
5 & $.91^{*}$ & .02 & $.73^{*}$ & -.01 & $.85^{*}$ & $.67^{*}$ \\
6 & $.83^{*}$ & $.65^{*}$ & $.95^{*}$ & .16 & $.82^{*}$ & $.86^{*}$ \\
7 & $.79^{*}$ & .54 & $.69^{*}$ & .03 & $.96^{*}$ & $.87^{*}$ \\
8 & $.74^{*}$ & $.65^{*}$ & $.70^{*}$ & $.71^{*}$ & $.76^{*}$ & $.82^{*}$ \\
9 & $.84^{*}$ & .10 & $.90^{*}$ & .44 & $.95^{*}$ & $.65^{*}$ \\
10 & $.90^{*}$ & .43 & $.92^{*}$ & $.69^{*}$ & $.52^{*}$ & $.79^{*}$ \\
Mean & .79 & .42 & .79 & .36 & .80 & .78 \\
\hline
\end{tabular}

*Statistically significant, $\alpha=.05$ (single-tailed test of positive correlation).

(mean presentation duration of $500 \mathrm{msec}$, range $=250-750 \mathrm{msec}$ ), 15 to both the left display and the right display and 20 to the center display. Performance was measured by a formula score that summed three quotients, one for each of three groups of target stimuli. Each quotient was computed by adding the number of correct responses, subtracting error responses, and dividing by the mean response latency. The original SEDI task is described by Mills and Bisgrove (1983). In the present experiment, the three displays were arranged in a planar configuration, and the center display was $60 \mathrm{~cm}$ directly in front of the subject's head, which was supported by a head-and-chin rest. The peripheral displays were located at an eccentricity of $20^{\circ}$.

Each subject received a total of $2 \mathrm{~h}$ of training on the IVR, pursuitrotor, and SEDI tasks prior to participation in the experimental sessions. The training session included five administrations of the IVR task battery, ten 50 -sec practice trials on the pursuit-rotor task, and 10 SEDI practice trials. The pursuit-rotor and SEDI practice constituted refresher training for the subjects, who had each completed 49 SEDI trials and 31 pursuit-rotor trials during previous studies.

Dosing. Each subject was asked to consume four drinks (one during each of four consecutive 10 -min drinking periods) at two separate times (drinking sessions started 60 min prior to Trials 3 and 6) on both testing days. In one testing session, the drinks contained a significant alcohol dose; in the other session, placebo drinks (a few milliliters of alcohol floated on top of orange, cranberry, and lime juices with tonic water) were served. Neither the subjects nor the task administrators were informed of the dosing conditions (doubleblind procedures). Four subjects received their alcohol doses during the first experimental session; the other 3 subjects received theirs during the second session. The alcohol doses were individually calculated on the basis of total body water derived from equations provided by Watson (1989). Specific procedures for drink preparation and administration are provided in Mundt, Perrine, and Searles (1997). The mean dose provided in each drinking period was $63.7 \mathrm{~g}$ of ethanol (range $=55.0-74.7 ; S D=6.2$ ), which converted to a mean of $0.76 \mathrm{~g} / \mathrm{kg}$ (range $=0.71-0.81 ; S D=0.04$ ). The 6 -h delay between the first and second drinking periods was designed to allow dosed subjects to approach a BAC of $0 \mathrm{mg} / \mathrm{dL}$ just prior to consumption of the second dose, producing two distinct $\mathrm{BAC}$ curves that were similar to one another.

Meals. The subjects were given a standard breakfast of three slices of French toast and an 8-oz cup of juice, and they were offered a cup of decaffeinated coffee at 7:30 Saturday morning. Between Trials 3 and 4, they were given a lunch consisting of a ham and turkey sandwich, carrot and celery sticks, and an 8-oz glass of noncaffeinated soda or juice. Both groups began lunch $1.5 \mathrm{~h}$ after their first drinking period and finished lunch $3.5 \mathrm{~h}$ before their second drinking period. Between Trials 6 and 7, the subjects were provided a meal of their choice from a local Chinese restaurant. At this time, they were also permitted another cup of decaffeinated coffee. After finishing their evening meal and throughout the remainder of the session, snack foods and noncaffeinated beverages were made available. An additional cup of decaffeinated coffee was permitted after 3:00 a.m. during the overnight part of the session.

Other. Between testing trials, the subjects stayed in the lounge area, where they consumed the drinks and meals. The subjects engaged themselves in reading, watching sports and videotapes on television, playing games, and conversation. Smoking was permitted in the lounge. They were not allowed to sleep; when presenting evidence of drowsiness during the overnight part of the session, they were roused by research staff to help keep them awake. Periodically, the subjects were allowed brief, supervised walks outside to stretch their legs and get fresh air. They were also permitted a shower during the early morning hours.

\section{Results}

Experiment 2 investigated whether performance measured by the IVR-administered assessment battery would reflect impairment induced by alcohol and/or fatigue. The mean BACs (average of entering and exiting measurements) of the subjects on the day they received alcohol across the 13 trials are shown in Figure 3. The mean BACs of Trials 3 and 6 (12-1 and 6-7 p.m.), Trials 4 and 7 (2-3 and 8-9 p.m.), and Trials 5 and 8 (4-5 and 10-11 p.m.) were 79,50 , and $16 \mathrm{mg} / \mathrm{dL}$, respectively. All other measurements were $0 \mathrm{mg} / \mathrm{dL}$, with the exception of 1 subject who had a reading of $7 \mathrm{mg} / \mathrm{dL}$ entering Trial 9.

Two previously validated tasks sensitive to the impairing effects of alcohol-the pursuit-rotor and SEDI taskswere performed in addition to the IVR tasks (e.g., Fillmore \& Vogel-Sprott, 1995; Ogden, Cairns, \& Curry, 1995). Initial ANOVAs, treating the alcohol/placebo testing order as a grouping variable, did not reveal statistically significant effects or interactions involving this factor. This difference between the subject groups was collapsed, and the influence of alcohol and fatigue on these tasks was assessed in 2 (dosing conditions) $\times 13$ (testing trials) repeated measures ANOVAs. Greenhouse-Geisser adjustments to the degrees of freedom were used because of indications of sphericity violations in the error components of the repeated factors. Figure 4 presents the mean performances on both the pursuit-rotor task (top panel) and the SEDI task 


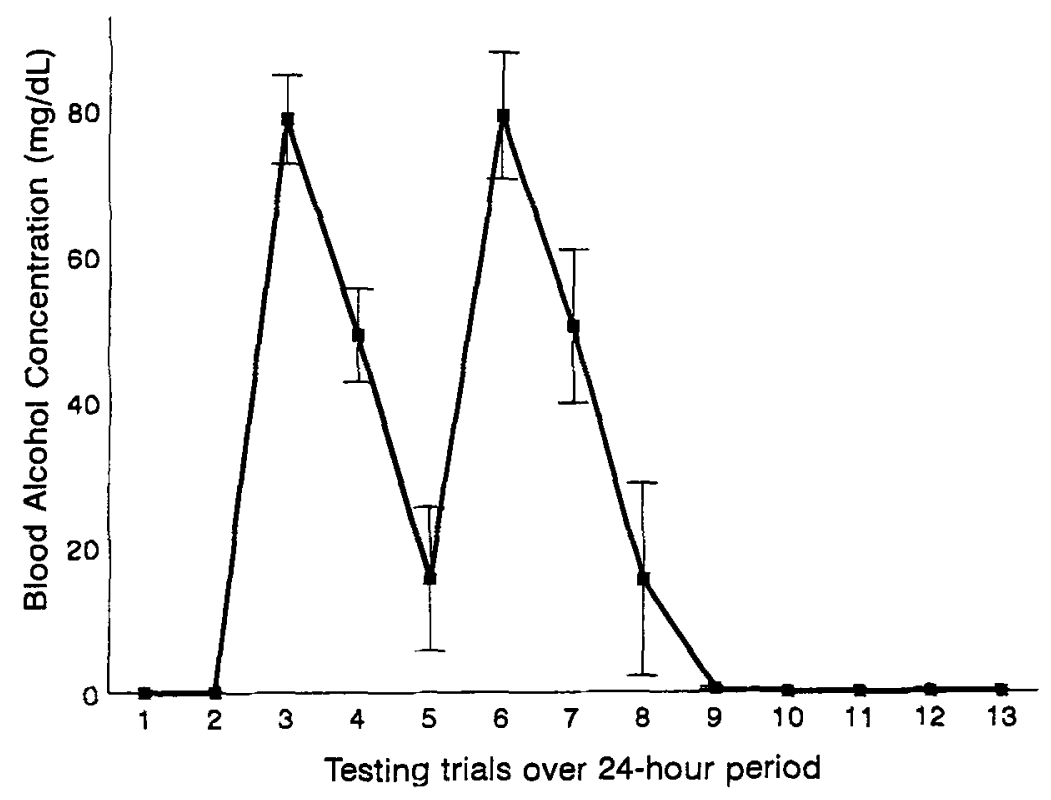

Figure 3. Mean blood alcohol concentrations $( \pm 1 S D$ ) of the 13 trials. Drinking sessions occurred prior to Trials 3 and 6 , ending 30 min prior to the beginning of these trials.

(bottom panel) on both the alcohol and the placebo consumption days. For the pursuit-rotor task, the main effect for dosing condition was marginally significant $[F(1,6)=$ $4.75, p=.07]$, and the main effect across trials was significant $[F(12,72)=4.12, p<.05]$, as was the interaction between these factors $[F(12,72)=3.68, p=.05]$. For the SEDI task, the main effect of dosing condition did not approach statistical significance $[F(1,6)=0.81]$; however, the main effect across trials was significant $[F(12,72)=$ $2.87, p<.05]$, and the interaction between these factors was marginally significant $\{F(12,72)=2.84, p=.054]$.

The trial effects for both the alcohol and the placebo conditions were analyzed separately in one-way ANOVAs across the 13 trials. In the pursuit-rotor task, the simple main effect across trials was significant on the alcohol day $[F(12,72)=4.37, p<.05]$, but only marginally significant on the placebo day $[F(12,72)=2.64, p=.092]$. Likewise, the trial effect was significant in the SEDI performance on the alcohol day $[F(12,72)=2.85, p<.05]$, and marginally significant on the placebo day $[F(12,72)=2.86$, $p=.062]$. The dosing condition $\times$ trial interactions were further analyzed by matched $t$ tests of the performances obtained at each of the trials on the alcohol and placebo testing days. Significant differences, indicated by asterisks in Figure 4, were obtained in the pursuit-rotor data for Trials 3,6 , and $7[t(6)=5.83,13.20$, and 3.06 , respectively]. Although the difference in means between dosing conditions appears larger at Trials 8-12 than the difference at Trial 7, the direction of the effect was not consistent across the individual subjects. SEDI performances differed significantly at Trials 3 and $6[t(6)=2.87$ and 2.53 , respectively].

The performances on the IVR-administered tasks were analyzed similarly to the pursuit-rotor and SEDI data, as- sessing first the between-subjects differences of session testing order and then the within-subjects factors of dosing condition and trials. The only session order effects in any of the mixed-model ANOVAs ( 2 groups $\times 2$ dosing conditions $\times 13$ trials) were session order $\times$ dosing condition interactions in the analyses of the half task and digit-span task $[F(1,5)=8.88$ and 9.05 , respectively, $p s<.05]$. These interactions were analyzed in separate ANOVAs of the alcohol/placebo performances for each group. Results of the digit-span analyses indicated marginally poorer performance during the alcohol session among the 4 subjects receiving alcohol during the first testing session $(M \mathrm{~s}=$ 6.85 and 7.52 , for the alcohol and placebo sessions, respectively) $[F(1,3)=9.3, p=.055]$. No difference was evident between the dosing conditions for the other 3 subjects $(M \mathrm{~s}=7.28$ and 7.05 , for the alcohol and placebo sessions, respectively) $[F(1,2)=1.69, p=.32]$. The analyses of the half task indicated an increase in performance from the first to the second testing session for both groups of subjects $[F(1,3)=4.76$, and $F(1,2)=12.76$, respectively]. These differences are not statistically significant ( $p$ s $>.05$ ). The increase in the number of correct responses (Ms $=3.5$ and 2.4, for the alcohol/placebo and placebo/ alcohol groups, respectively) probably reflects a learning carryover effect from the first to the second testing session.

The mean task performances across the 13 trials for both the alcohol and placebo testing sessions are shown in Figure 5 (note change in ordinate scale from Figure 1 ). Each task was analyzed in 2 (dosing conditions) $\times 13$ (trials) repeated measures ANOVAs. The results of the memory-probe task indicated no significant effects or interactions, reflecting the same performance ceiling effect found in Experiment 1. In addition, the analysis of the 

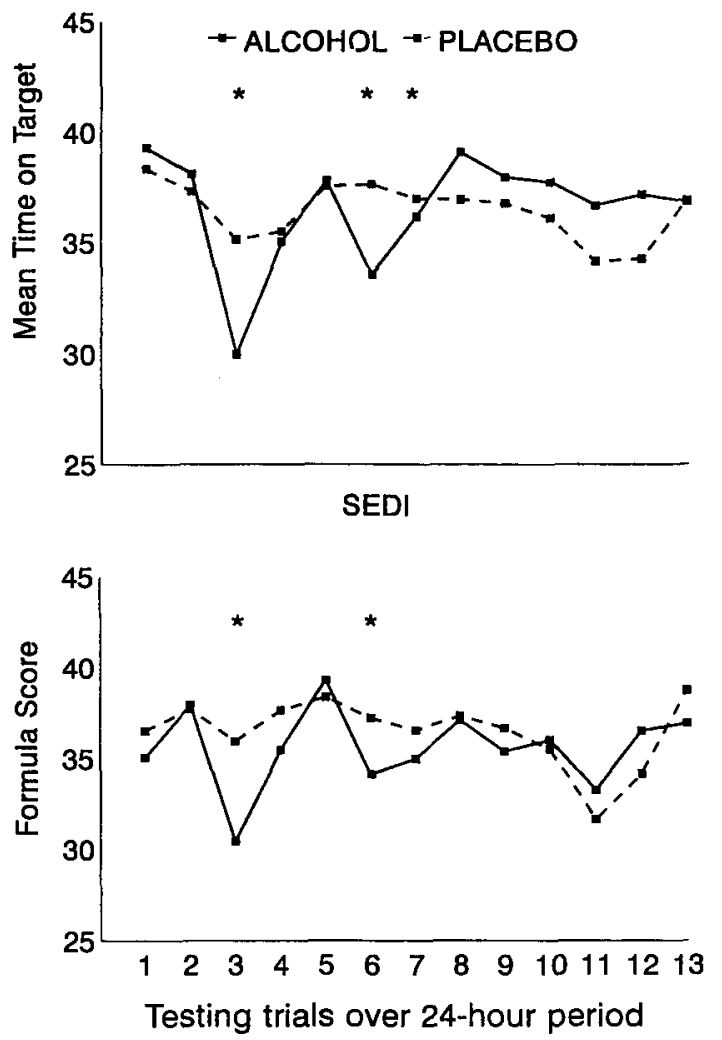

Figure 4. Top panel: Average time on target during two consecutive 50-sec runs on a photoelectric pursuit-rotor tracking a circular stimulus at 30 rpm across 13 trials. Bottom panel: Mean SEDI formula score (combined score representing speed and accuracy of responses to both peripheral and central stimuli) of the 2.5-min SEDI task.

one-back task did not reveal systematic dosing condition or trial effects. The analysis of the half task indicated a marginally significant trial effect over the 24-h duration of the sessions $[F(12,72)=2.67, p=.058]$, reflecting the general increase in performance from Trials 1-8 (commensurate with learning) and more erratic performances obtained during the early morning trials (midnight to 5 a.m.). The analyses of the digit-span, ordering, and follow-the-leader tasks did indicate performance impairment due to alcohol consumption. Analyses of dosing condition $X$ trial interactions reflected an influence of alcohol on performance $[F \mathrm{~s}(12,72)=1.73,2.18$, and 2.14 , respectively] but failed to attain statistical significance when the Greenhouse-Geisser adjustments to the degrees of freedom were applied ( $p \mathrm{~s}=.17, .12$, and .10 , respectively). Given the small sample size of this experiment, this adjustment may be excessively conservative since less stringent adjustments (Huynh-Feldt) indicated that the differences approached or exceeded statistical significance criteria. In fact, even with this conservative adjustment, when the alcohol and placebo sessions were analyzed separately, the main effect across trials on the alcohol day was significant for the follow-the-leader task $[F(12,72)=2.97$, $p<.05]$ and marginally significant for the ordering task $[F(12,72)=3.14, p=.06]$. The simple main effect across trials did not approach significance during the placebo session for either of these tasks, nor for either session of the digit-span task.

Similar to the analyses of the pursuit-rotor and SEDI tasks, matched $t$ tests were performed comparing the alcohol and placebo performances at each of the trials. These analyses indicated significant impairment in the alcohol session relative to the placebo session in Trial 3 for the ordering, digit-span, and follow-the-leader tasks $[t \mathrm{~s}(6)=$ $3.44,3.62$, and 3.58 , respectively, $p s<.05]$. In addition, performance in the one-back task at Trial 12 on the alcohol session was significantly worse than the corresponding performance on the placebo session $[t(6)=3.02, p<.05]$. These differences are noted by asterisks in Figure 5.

Two additional analyses were conducted to assess the sensitivity of the IVR-administered tasks to the impairing effects of alcohol and fatigue. Since the univariate analyses of the ordering, digit-span, and follow-the-leader tasks indicated sensitivity to the effects of alcohol, at least at the initial peak BAC (Trial 3), the performances of these tasks were combined into a single measure. The mean of Trials 1 and 2 , across both testing days, was computed as an individual baseline level of performance, and the performances at each trial were divided by this value. The baseline percentages for the three tasks were averaged and entered into a 2 (dosing conditions) $\times 13$ (trials) repeated measures ANOVA. A significant dosing condition $\times$ trial interaction was found $[F(12,72)=3.44, p<.05]$. Matched $t$ tests comparing the alcohol and placebo performances at each of the trials indicated a significant difference at Trial $3[t(6)=6.22, p<.001]$. These results are shown in the top panel of Figure 6.

An analysis of error responses produced somewhat more interesting results. There were generally too few errors on any given task at the various trials to analyze meaningfully; however, summing the total number of errors across all of the tasks (except the digit-span task, which measured performance until an error was produced) produced consistent effects. These results are shown in the bottom panel of Figure 6. An ANOVA indicated a significant dose $\times$ trial interaction $[F(12,72)=3.24, p<.05]$, reflecting significantly more errors at both peak BAC trials (Trials 3 and 6) and at Trial $12[t \mathrm{~s}(6)=3.40,5.30$, and 2.46 , respectively, $p \mathrm{~s}<.05$ ], relative to performances obtained during the placebo session.

\section{Discussion}

The results of Experiment 2 clearly indicate promise for IVR-administered psychological performance assessment. However, procedural refinements and broader task demands are needed in future implementations to increase the sensitivity of such test batteries. Given the small sample size of this experiment and the number of analyses and multiple comparisons performed, the results must be viewed cautiously. While conservative adjustments (Greenhouse- 
Ordering Task

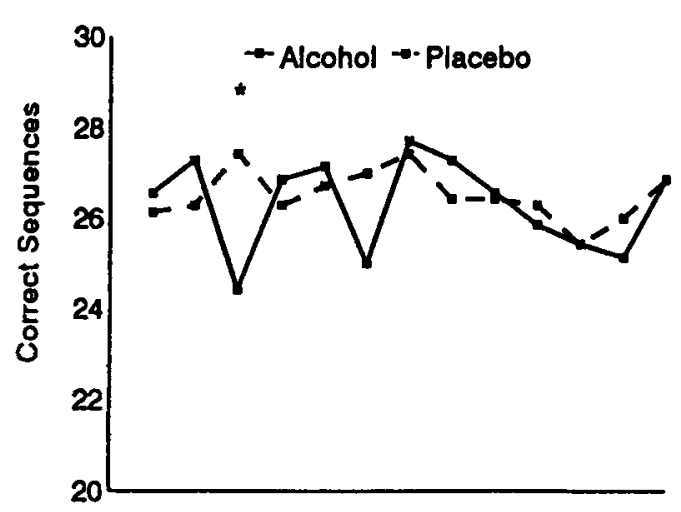

Memory Probe

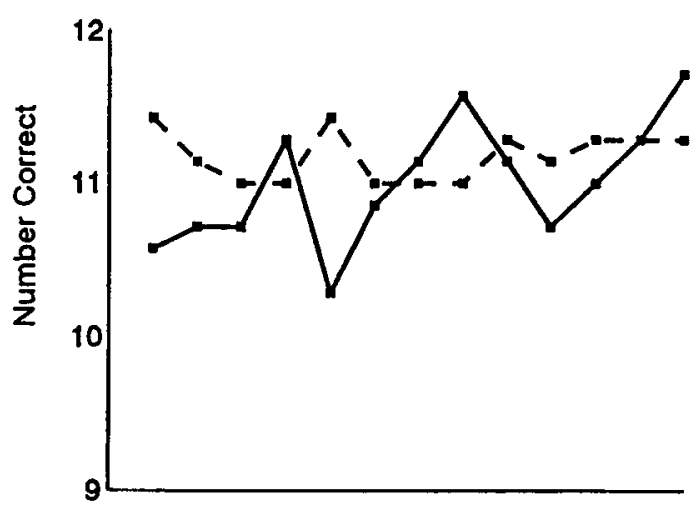

Digit Span

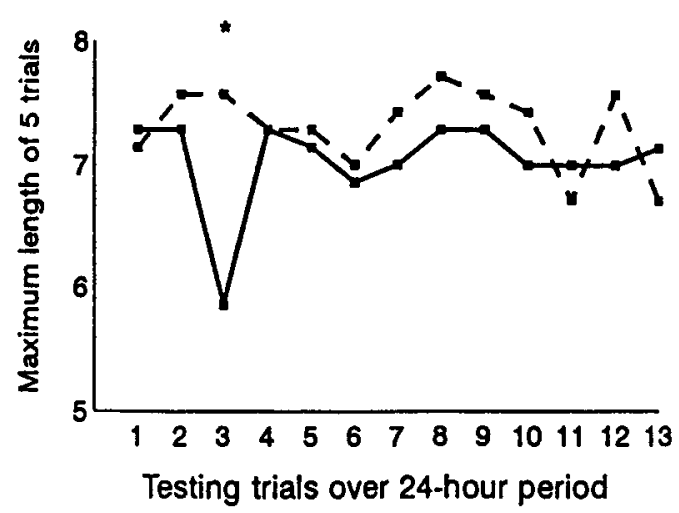

One Back Task

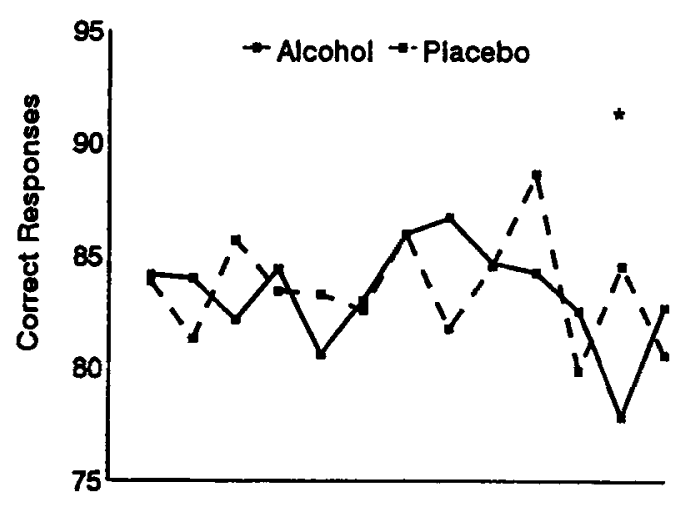

Follow the Leader

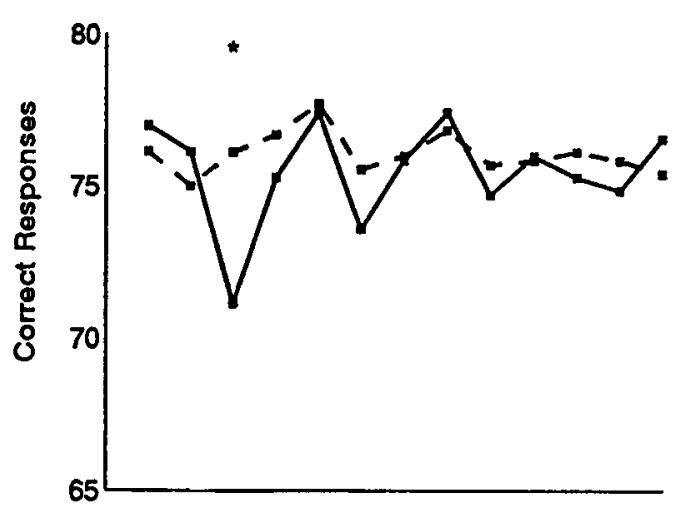

Half Task

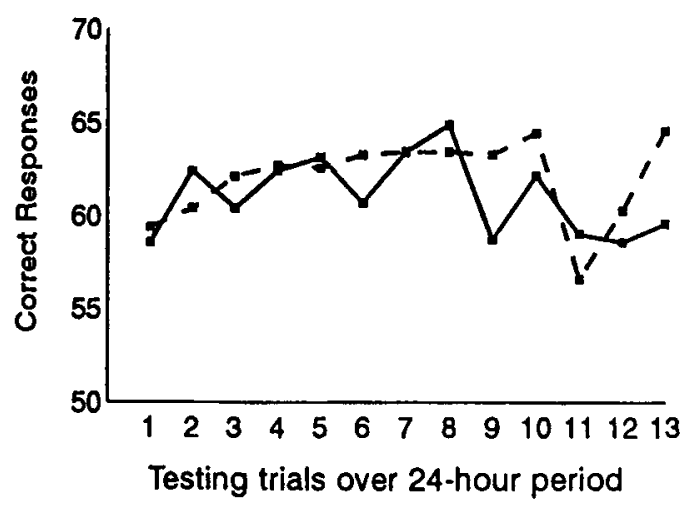

Figure 5. Alcohol and placebo session task performances of the six IVR-administered tasks across the 13 trials of both 24-h sessions. Significant differences in trial performances between the two dosing conditions are indicated by asterisks.

Geisser) were used for the inferential probability values of the omnibus $F$ statistics reported, and the paired comparisons reported as significant were two-tailed tests (despite anticipation of alcohol-related impairment), the probability of Type I error was undoubtedly increased. All of the comparisons reported as statistically significant, however, were in expected and readily interpretable direc- tions, reducing the likelihood that they simply reflect spurious outcomes that capitalized on chance.

Analyses of the pursuit-rotor and SEDI tasks, included in this experiment to assess the convergent validity of the IVR-administered tasks, indicate that the subjects were impaired at Trials 3 and 6, with less impairment evident at the latter trial despite equivalent BACs. The diminished 
response over time at equivalent BACs reflects acute alcohol tolerance effects, a topic of considerable research interest but beyond the scope of this paper (see Chesher \& Greeley, 1992, Martin \& Moss, 1993, Radlow, 1994, and Vogel-Sprott, 1979, for extensive discussions of acute alcohol tolerance). The data obtained from these validated tasks, specifically designed to be maximally sensitive to alcohol impairment, indicate that the influence of alcohol and fatigue on the performances in the present experiment was not overwhelming. Three of the IVR-administered tasks - follow the leader, ordering, and digit span - did detect impaired performance at Trial 3, and the analysis of response errors indicated impairment at Trials 3 and 6.

These results are quite encouraging. Future enhancements of these tasks and expansion of the assessment battery are likely to produce better convergence with previously validated instruments. Future refinements of IVR-based performance assessment may someday rival the sensitivity and specificity of current laboratory-based performance measures.

Clearly, some of the tasks, such as the memory probe, indicate marginal reliability or sensitivity that may be due to performance ceiling effects. While greater perturbation of the subjects would likely produce degraded performance, it would also be relatively easy to increase task difficulty by (1) increasing the size of the presented memory sets, (2) presenting the memory sets in less time (i.e., speeding up presentation of the memory set sequence), or (3) using more trials in the task battery. Analyses of performance on two other tasks - the one-back and half tasks-also indicated relative insensitivity to the detrimental effects of alcohol. Examination of the data in Figure 5, however, indicates that they may hold the greatest potential for assessing fatigue effects. During the early morning hours, between midnight and 5 a.m. (Trials 9-11), performance on these tasks, along with the ordering task, appears to decline and show greater instability. Interestingly, these tasks are the most cognitively demanding of the assessment battery in terms of their reliance on conscious, controlled processing (Shiffrin \& Schneider, 1977). Responses in the half task are variably mapped (i.e., a correct response to any given stimulus presentation is variable), the one-back performance requires effortful inhibition of a natural inclination to respond immediately to the most recently presented digit, and the ordering task requires mental rearrangement of the presented sequence prior to responding. Perhaps not surprisingly, performance on these tasks also reflected the greatest influence of learning during training in Experiment 1 . While speculative at this point, the data of Experiment 2 suggest that refinement of these tasks, or development of tasks assessing similar cognitive functions, is most likely to yield results sensitive to performance impairment due to fatigue.

The results of Experiment 2 also suggest that the tasks requiring less cognitive processing, such as follow the leader, are more sensitive to the impairing effects of alcohol. It may well be that increased sensitivity will be obtained from even greater task simplification. Perhaps the use of a more restricted digit set (e.g., 1,3,7,9 rather than $1-9$, inclusive)
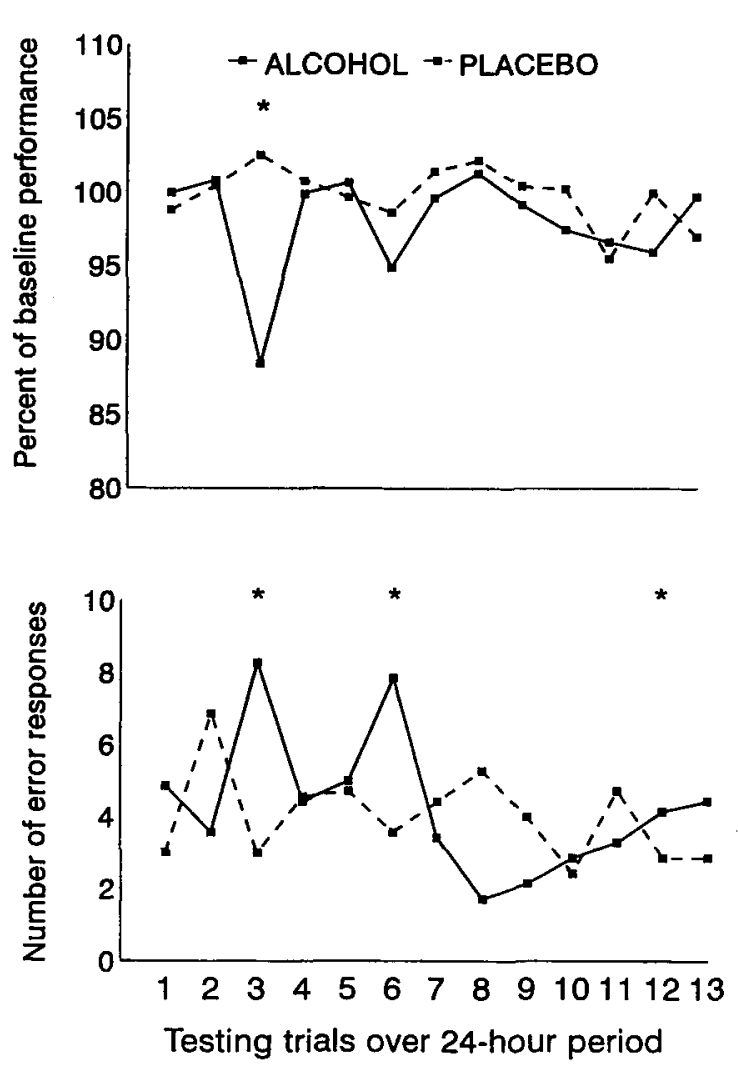

Figure 6. Top panel: Mean percentage of baseline performance for combined follow-the-leader, ordering, and digit-span performances. Baseline performance was operationally defined as the average performance of Trials 1 and 2 across both testing sessions. Bottom panel: Average number of error responses summated over five tasks (digit-span task excluded). Significant differences in trial performances between the two dosing conditions are indicated by asterisks.

would increase both the reliability and sensitivity of such tasks. Maylor, Rabbitt, James, and Kerr (1992) found that reaction times to two-choice tasks were more sensitive to the effects of alcohol than were reaction times to four- to eightchoice tasks. Simple psychomotor "tapping" tasks (Kennedy, Dunlap, Turnage, \& Fowlkes, 1993), requiring open-looped, speeded motor responses, may also hold promise.

Confirmation or rejection of such speculations, however, must await further refinement and independent assessment of the methodology. The results of Experiment 2 are promising for such developments, but they are presently little more than seedlings with considerable growth potential. Extensive additional research and reiterative task development are needed to cultivate these initial investigations of IVR-based testing into a reliable and validated assessment methodology.

\section{GENERAL DISCUSSION}

These initial experiments were designed to assess the feasibility and potential applicability of IVR technology to the distal assessment of psychological functioning. To 
our knowledge, this study is the first such endeavor, and the results suggest that such an approach holds promise for future research and development. The unbridled growth in PC-based software and hardware has been a boon to psychological research in the laboratory. However, bringing together the experiment populations of interest and the technology for assessment often presents difficulties and practical limitations. Furthermore, important situational contexts or other circumstances that might be critical to performance may be difficult or impossible to replicate in typical testing protocols. A performance-based assessment methodology as close as the nearest telephone may obviate research barriers that have previously been impenetrable. Such applications also present technical challenges regarding positive user identification and assurance that aides, such as paper-and-pencil records, are not used to assist performance.

The telephone system infrastructure is largely in place, with the technical and informational capacities of this system expanding rapidly (touch-tone service is presently available in $90 \%-95 \%$ of the Central Offices). The deceptively simple telephone interface between individual and technology invokes little apprehension among the population at large. What remains is the refinement and more complete assessment of the methodology suggested by the present research.

Before ushering in a new age of telephony-based performance assessment batteries, however, substantial foundational work remains. The tasks of the present study were educated guesses and derivations of established tasks that were thought to hold potential for IVR administration. While the results of this study are encouraging for further development, they are far from definitive evidence establishing practical utility of such a methodological approach. The glaring issue of marginal sensitivity in the present data aside, broader issues that could not be addressed by the present research await future investigation. Further refinements and extensions of the tasks used were briefly discussed above, and the development of other tasks assessing semantic or grammatical reasoning are easily conceivable. Addressing the extent to which such tasks assess common or unique dimensions of psychomotor and cognitive functioning would require much larger sample sizes than those of the present study. It would be hoped, however, that a rich factor structure, reflecting assessment of diverse psychological functions, would result from analysis of more extensive data sets.

Hardware issues are likely to affect future research in this area. The spatial layout of keys on telephone keypads has been standardized; however, other aspects of the personmachine interface vary considerably. The size and distance between keys, as well as resistance and rebound coefficients of the button mechanisms, should be expected to influence performance. Obviously, telephones with the keypad imbedded in the handset cannot be used. These issues could easily be addressed by the production of standardized interfaces that are attached to existing telephones or plugged directly into the modular telephone jack. Such devices could standardize the acoustical properties of the information heard by subjects and address human-factors issues concerning response execution.

A more substantive issue regarding the proposed methodology that will require considerable investigation concerns the theoretical and practical equivalence between apparently parallel forms of assessment. While Kennedy et al. (1989) found that auditory and visual implementations of a counting task appeared to tap identical mental processes, differences in the mean scores were found. Performance on more complex tasks may well be influenced by differences in visual and auditory encoding of information and subsequent processing of information toward response selection and execution. Multiple resource theory (Wickens, 1984) suggests that the sensory modality for presenting information has important implications for response compatibility. Visually presented spatial information is most compatible with manual responses, whereas verbal information is more compatible with vocal responses. These considerations may be crucial for current IVR applications dependent on manually executed keypresses, and they could influence future implementations using voice recognition technology (which may also assist with user recognition) as it becomes increasingly robust to idiosyncratic differences between speakers. Resolution of these issues will likely require substantial research using special subject populations with known neuropsychological characteristics (e.g., stroke victims, dementia patients, patients with Parkinson's disease, etc.). The validity of the proposed methodology can then be assessed with respect to validated assessment instruments with established population norms.

These initial experiments indicate that performance assessment via interactive voice response technology is feasible and suggest that such an approach may hold potential for clinical and commercial applications. Remote assessment of fitness-for-duty, longitudinal assessment of disease progression and/or pharmaceutical efficacy, and broadly accessible assessment of psychological functioning using standardized computer-administered testing procedures may follow the basic research and development needed to validate and establish this methodology. Acceptance of the approach, and ultimately the utility of the applications developed, will depend on the scientific rigor and soundness of the methods used to evaluate the developmental progress.

\section{REFERENCES}

American Psychological Association (1989). Ethical principles in the conduct of research with human participants. Washington, DC: Author.

Baer, L., Brown-Beasley, M. W., Sorce, J., \& Henriques, A. I. (1993). Computer-assisted telephone administration of a structured interview for obsessive-compulsive disorder. American Journal of Psychiatry, 150, 1737-1738.

Baer, L., Jacobs, D. G., Cukor, P., O'Laughlen, J., Coyle, J. T, \& MAGRUDER, K. M. (1995). Automated telephone screening survey for depression. Journal of the American Medical Association, 273, $1943-$ 1944. 
Chesher, G., \& Greeley, J. (1992). Tolerance to the effects of alcohol. Alcohol, Drugs \& Driving, 8, 93-106.

ElSMORE, T. F. (1994). SYNWORK1: A PC-based tool for assessment of performance in a simulated work environment. Behavior Research Methods, Instruments, \& Computers, 26, 421-426.

FillmoRE, M. T., \& VoGEL-SPRoTT, M. (1995). Expectancies about alcohol-induced motor impairment predict individual differences in responses to alcohol and placebo. Journal of Studies on Alcohol, 56, 90-98.

Greist, J. H., Gustafson, D. H., Stauss, F. F., Rowse, G. L., LaughREN, T. P., \& Chiles, J. A. (1973). A computer interview for suicide-risk prevention. American Journal of Psychiatry, 30, 1327-1332.

GREIST, J. H., \& KLEIN, M. H. (1980). Computer programs for patients, clinicians, and researchers in psychiatry. In J. B. Sidowski, J. H. Johnson, \& T. A. Williams (Eds.), Technology in mental health care delivery systems (pp. 161-181). Norwood, NJ: Ablex.

Greist, J. H., KLEIN, M. H., \& VAN CURA, L. J. (1973). A computer interview for psychiatric patient target symptoms. Archives of General Psychiatry, 29, 247-253.

Greist, J. H., VAN CuRA, L. J., \& KNEPPRETh, N. P. (1973). A computer interview for emergency room patients. Computers \& Biomedical Research, 6, 257-265.

Kennedy, R. S., Baltzley, D. R., Wilkes, R. L., \& Kuntz, L. A. (1989). Psychology of computer use: IX. A menu of self-administered microcomputer-based neurotoxicology tests. Perceptual \& Motor Skills, 68, 1255-1272.

Kennedy, R. S., Dunlap, W. P., Turnage, J. J., \& Fowlkes, J. E. (1993). Relating alcohol-induced performance deficits to memory capacity: A suggested methodology. Aviation, Space, \& Environmental Medicine, 64, 1077-1085.

MARTIN, C. S., \& Moss, H. B. (1993). Measurement of acute tolerance to alcohol in human subjects. Alcoholism: Clinical \& Experimental Research, 17, 211-216.

Maylor, E. A., Rabbitt, G. H., James, G. H., \& Kerr, S. A. (1992). Effects of alcohol, practice, and task complexity on reaction time distrjbutions. Quarterly Journal of Experimental Psychology, 44A, 119-139.

McKnight, A. S., \& McKnight, A. J. (1994). The Automated Psychophysical Test (APT) for assessing age-diminished capabilities. Behavior Research Methods, Instruments, \& Computers, 26, 187-191.

MiLls, K. C., \& BisGrove, E. Z. (1983). Cognitive impairment and perceived risk from alcohol: Laboratory, self-report, and field assessments. Journal of Studies on Alcohol, 44, 26-46.

Mundt, J. C., Perrine, M. W., \& Searles, J. S. (1997). Individual differences in alcohol responsivity: Physiological, psychomotor, and subjective response domains. Journal of Studies on Alcohol, 58, 130140.

Mundt, J. C., Perrine, M. W., Searles, J. S., \& Walter, D. (1995). An application of interactive voice response (IVR) technology to longitudinal studies of daily behavior. Behavior Research Methods, Instruments, \& Computers, 27, 351-357.
Mundt, J. C., Searles, J. S., Perrine, M. W., \& Helzer, J. E. (1995) Cycles of alcohol dependence; Frequency-domain analyses of daily drinking logs for matched alcohol-dependent and nonclinical subjects. Journal of Studies on Alcohol, 56, 491-499.

National Advisory Council on Alcohol Abuse and Alcohol ISM (1989). Recommended council guidelines on ethyl alcohol administration in human experimentation. Washington, DC: National Institute on Alcohol Abuse and Alcoholism.

Ogden, E. J. D., Cairns, I., \& CURRY, E. (1995). Impairment of learning by low dose alcohol. In C. N. Kloeden \& A. J. McLean (Eds.), Alcohol, drugs and traffic safety-T95 (pp. 627-632). Adelaide, Australia: NHMRC Road Accident Research Unit, University of Adelaide.

Perrine, M. W., Mundt, J. C., Searles, J. S., \& Lester, L. S. (1995). Validation of daily self-reported alcohol consumption using interactive voice response (IVR) technology. Journal of Studies on Alcohol, 56, $487-490$

Psychological Corporation (1996). Catalog: Tests and related products for psychological assessment. San Antonio, TX: Harcourt Brace.

RADLOw, R. (1994). A quantitative theory of acute tolerance to alcohol. Psychopharmacology, 114, 1-8.

Richards, J. S., Fine, P. R., Wilson, T. L., \& Rogers, J. T. (1983). A voice-operated method for administering the MMPI. Journal of Personality Assessment, 47, 167-170.

Searles, J. S., Perrine, M. W., Mundt, J. C., \& Helzer, J. E. (1995). Self-report of drinking by touch-tone telephone: Extending the limits of reliable daily contact. Journal of Studies on Alcohol, 56, 375-382.

ShIFFrin, R. M., \& SCHNEIDER, W. (1977). Controlled and automatic human information processing: II. Perceptual learning, automatic attending, and a general theory. Psychological Review, 84, 127-190.

Turnage, J. J., \& Kennedy, R. S. (1992). The development and use of computerized human performance test battery for repeated-measures applications. Human Performance, 5, 265-301.

Turnage, J. J., Kennedy, R. S., Smith, M. G., Baltzley, D. R., \& LANE, N. E. (1992). Development of microcomputer-based mental acuity test. Ergonomics, 35, 1271-1295.

Vogel-SPROTT, M. D. (1979). Acute recovery and tolerance to low doses of alcohol: Differences in cognitive and motor skill performance. $P$ sy chopharmacology, 61, 287-291.

VOGEL-SPROTT, M. D. (1992). Alcohol tolerance and social drinking: Learning the consequences. New York: Guilford.

WATSON, P. E. (1989). Total body water and blood alcohol levels: Updating the fundamentals. In K. E. Crow \& R. D. Batt (Eds.), Human metabolism of alcohol: Vol. 1. Pharmacokinetics, medicolegal aspects and general interests (pp. 41-59). Boca Raton, FL: CRC.

WICKENS, C. D. (1984). Engineering psychology and human performance. Columbus, $\mathrm{OH}$ : Charles E. Merrill.

(Manuscript received May 23, 1996; revision accepted for publication April 7, 1997.) 